\title{
SIRT6 regulates the proliferation and apoptosis of hepatocellular carcinoma via the ERK1/2 signaling pathway
}

\author{
CUISHENG ZHANG ${ }^{1 *}$, YING YU $^{2 *}$, QINGXIAN HUANG ${ }^{1}$ and KUN TANG ${ }^{1}$ \\ Departments of ${ }^{1}$ Hepatobiliary Surgery and ${ }^{2}$ Vascular Surgery, \\ Yuhuangding Hospital of Yantai, Yantai, Shandong 264000, P.R. China
}

Received September 10, 2018; Accepted May 20, 2019

DOI: $10.3892 / \mathrm{mmr} .2019 .10398$

\begin{abstract}
Hepatocellular carcinoma (HCC) is the most common type of liver cancer, and exhibits a high mortality rate. Sirtuin (SIRT)6 is a member of the sirtuin family, which may be useful targets in the treatment of tumors. The present study aimed to explore the expression of SIRT6 in numerous HCC cell lines and investigate the role of SIRT6 in the proliferation and apoptosis of the HCC cells, and the underlying mechanisms. Overexpression and silencing of SIRT6 were performed by transfection of Huh-7 cells with synthetic overexpression and small interfering RNA (siRNA) plasmids. Cell proliferation was evaluated using a Cell Counting Kit-8 assay. The apoptosis rate was measured via flow cytometry. Cloning efficiency was assessed using plate clone formation assays. The expression of mRNAs and proteins were determined via reverse transcription-quantitative PCR and western blot analyses, respectively. SIRT6 was overexpressed in Hep3B, Huh-7, MHCC-97H, MHCC-97L, MHCC-LM6, MHCC-LM3, YY-8103 and SK-hep-1 cell lines, compared with MIHA and HL-7702 normal liver cell lines. Overexpression of SIRT6 increased the proliferation of Huh-7 cells, upregulated the expression of $\mathrm{Bcl}-2$ and phosphorylation of extracellular-signal regulated protein kinase (ERK), and decreased the expression of cleaved-caspase-3 and Bcl-2-associated X protein (Bax) in Huh-7 cells. siRNA-mediated silencing of SIRT6 decreased the proliferation and increased the apoptosis of Huh-7 cells, downregulated the expression of Bcl-2 and phosphorylated-ERK, and promoted the expression of cleaved-caspase-3 and Bax. The proliferation of Huh-7 cells was decreased using the ERK1/2 inhibitor U0126. The results suggested that SIRT6 affected the proliferation and apoptosis of HCC cells via the
\end{abstract}

Correspondence to: Dr Kun Tang, Department of Hepatobiliary Surgery, Yuhuangding Hospital of Yantai, 20 Yudong Road, Zhifu, Yantai, Shandong 264000, P.R. China

E-mail: tangkun_kunta@163.com

*Contributed equally

Key words: sirtuin 6, hepatocellular carcinoma, ERK1/2, tumorigenesis, regulation regulation of the ERK1/2 pathway, altering the activation of the intrinsic apoptosis pathway. SIRT6 may be a potential target for the treatment of $\mathrm{HCC}$; however, its role requires further investigation.

\section{Introduction}

In total, $\sim 80 \%$ of primary liver cancers are hepatocellular carcinomas (HCCs) (1). The onset of HCC is concealed, and radiotherapy, chemotherapy and surgical treatment do not reliably improve the prognosis of patients, leading to a high mortality rate $(1,2)$. Malignant proliferation and escape from apoptosis are the most important pathological features of HCC (1,3). Therefore, studying the molecular mechanisms regulating the malignant proliferation and apoptosis of $\mathrm{HCC}$ are required for developing effective therapeutic interventions.

The sirtuin family are class III histone deacetylases of nicotinamide adenine dinucleotide (NAD+) (4). Previous studies have demonstrated that sirtuin family members regulate gene expression by deacetylating various non-histone proteins, contribute to various important physiological activities, including cell differentiation, apoptosis, aging and energy metabolism, and serve important roles in the formation and development of various tumors (5-8). Sirtuin (SIRT)6 is localized in nuclear heterochromatin, and exhibits adenosine diphosphate-ribosyltransferase and deacetylase activities (9). At present, the effects of SIRT6 on the proliferation and apoptosis of HCC remain unclear, and the functions of SIRT6 in promotion or inhibition during progression of tumor development are controversial (10); however, SIRT6 has exhibited the potential as a candidate target in the treatment of tumors $(11,12)$. Therefore, the study of SIRT6 in HCC is urgent and valuable.

Mitogen-activated protein kinase (MAPK) is an important transmitter of signal transduction from the cell membrane to the inner nucleus (13). Extracellular-signal regulated protein kinase (ERK) and c-Jun N-terminal kinase (JNK) are two subfamilies of MAPK; abnormal activation of these kinases is associated with the development of liver cancer (13). Phosphorylation of ERK can activate a variety of target molecules, including c-Jun, c-Fos and G1/S-specific cyclin-D1, promoting the development of liver cancer (14). Additionally, the JNK signaling pathway can promote the expression of downstream pathway proteins c-Jun, P53 and P21, induce the 
proliferation and angiogenesis of liver cancer, and contribute towards the regulation of the cell cycle and apoptosis of liver cancer cells (14). Therefore, the activation of the ERK1/2 signaling pathway may be a mechanism underlying the development of HCC.

The present study aimed to evaluate the expression of SIRT6 in various HCC cell lines, and investigate the role of SIRT6 in cell proliferation and apoptosis, further determining the involvement of the ERK1/2 signal pathway. The present study provided novel insight into the role of SIRT6 in HCC, and the potential underlying mechanisms.

\section{Materials and methods}

Cell culture, plasmid, grouping and transfection. HL-7702, MIHA, Hep3B, Huh-7, MHCC-97H, MHCC-97L, MHCC-LM6, MHCC-LM3, YY-8103, SK-hep-1 cell lines were all obtained from the Type Culture Collection of the Chinese Academy of Sciences. The HL-7702 cell line is a normal liver cell line, and was used as a control group. HL-7702 and MIHA cells were cultured in RPMI-1640 medium (Thermo Fisher Scientific, Inc.); Hep3B, Huh-7, MHCC-97H, MHCC-97L, MHCC-LM6, MHCC-LM3, YY-8103, SK-hep-1 cell lines were cultured in DMEM (Thermo Fisher Scientific, Inc.). Medium was supplemented with $10 \%$ fetal bovine serum (HyClone; GE Healthcare Life Sciences), and cells were cultured at $37^{\circ} \mathrm{C}$ with $5 \% \mathrm{CO}_{2}$. Cells were subcultured every 2-3 days. The SIRT6 overexpression plasmid, the negative control (NC) plasmid (empty pcDNA3.1 vector), the plasmid containing small interfering RNA against SIRT6 (siSIRT6) and the plasmid containing NC-siRNA were synthesized by Shanghai GenePharma Co., Ltd. The sequences of oligonucleotides were as follow: siSIRT6, 5'-TCATGACCCGGCTCA TGAA-3'; NC-siRNA, 5'-TCACCCATCGGTACGTGAA-3'. Huh-7 cell line was divided into 3 groups: Control (blank); NC or siNC (cells transfected with the NC plasmid or NC-siRNA plasmid); and SIRT6 overexpression or siSIRT6 groups (cells transfected with the SIRT6 overexpression or siSIRT6 plasmid). To further explore whether SIRT6 affects the proliferation and apoptosis of HCC cells by regulating the ERK1/2 pathway, the cells were treated with $10 \mu \mathrm{M} \mathrm{U} 0126$ at $37^{\circ} \mathrm{C}$ for $24 \mathrm{~h}$, and U0126 treatment and transfection occurred simultaneously. Huh-7 cells were also separated into control (blank), NC (cells transfected with the NC plasmid), SIRT6 (cells transfected with the SIRT6 overexpression plasmid), U0126 (cells treated with $10 \mu \mathrm{M}$ U0126), SIRT6 + U0126 (cells transfected with the SIRT6 overexpression plasmid and treated with $10 \mu \mathrm{M}$ U0126) groups for the measurement of cell proliferation using ERK1/2 inhibitor U0126. Transfection was conducted using Lipofectamine ${ }^{\mathrm{TM}} 2000$ transfection reagent (Invitrogen; Thermo Fisher Scientific, Inc.) according to the manufacturer's protocols. Following transfection for $48 \mathrm{~h}$, cells were used to subsequent experiments.

Cell proliferation assay. Cell proliferation was assayed using a Cell Counting Kit-8 (Sigma-Aldrich; Merck KGaA) at 0,24 and $48 \mathrm{~h}$ following transfection. Procedures were performed according to the manufacturer's protocols. Cells $\left(3 \times 10^{3}\right.$ cells/well) were then incubated in a 96 -well plate for $1 \mathrm{~h}$ at $37^{\circ} \mathrm{C}$, and the optical density (OD) was measured at $450 \mathrm{~nm}$ using a microplate reader $\left(\right.$ Multiskan $^{\mathrm{TM}}$; Thermo Fisher Scientific, Inc.).

Measurement of apoptosis via flow cytometry. Following transfection for $48 \mathrm{~h}$, Huh-7 cells were washed with PBS and dissociated with $0.25 \%$ trypsin for $2 \mathrm{~min}$ at $37^{\circ} \mathrm{C}$. Cells $\left(1-5 \times 10^{5}\right)$ were centrifuged at $800 \mathrm{x}$ g for $5 \mathrm{~min}$ and collected. Using an Annexin V-FITC Apoptosis Staining/Detection kit (Abcam), $195 \mu \mathrm{l}$ Annexin $\mathrm{V}$ binding solution, $6 \mu \mathrm{l}$ Annexin $\mathrm{V}$ and $4 \mu \mathrm{l}$ propidium iodide were added to cells, and then mixed gently by pipetting. Cells were incubated at room temperature for $20 \mathrm{~min}$ in the dark, and immediately analyzed using a flow cytometer (BD FACSCanto II; BD Biosciences) and FACSDiva software version 6.1.2 (BD Biosciences) to detect the apoptosis rate. Flow cytometry demonstrated that the advanced apoptotic cells were in the upper right quadrant, and the early apoptotic cells were in the lower right quadrant. The apoptotic rate was the sum of the early and advanced apoptotic rates.

Plate clone formation assay. Transfected cells were collected and inoculated into a $100-\mathrm{mm}$ dish at a concentration of 200 cells/dish following transfection for 48 h. G418 $(700 \mu \mathrm{g} / \mathrm{ml}$; Abcam) was added to the medium and mixed to detect positive cell clones for 3 weeks until visible cell clones emerged. Fresh medium was replaced every 3 days. The supernatant was then discarded, and the cells were gently washed with PBS twice. Cells were fixed with methanol for $15 \mathrm{~min}$ at room temperature, and stained with crystal violet for 10-30 min at room temperature. Cells were then washed slowly with running water and dried. Each cell clone on the dishes was counted and photographed under a light microscope (magnification, x40).

Assessment of $m R N A$ levels via reverse transcriptionquantitative $(R T-q) P C R$. Total RNA was extracted using TRIzol $^{\circledR}$ (Invitrogen; Thermo Fisher Scientific, Inc.) and $1 \mu \mathrm{g}$ of RNA of each sample was transcribed into cDNA using an iScript $^{\mathrm{TM}}$ cDNA Synthesis kit (Bio-Rad Laboratories, Inc.). The reverse transcription reaction was performed at $42^{\circ} \mathrm{C}$ for $15 \mathrm{~min}$, followed by reverse transcriptase inactivation at $85^{\circ} \mathrm{C}$ for 15 sec. A Fast Start Universal SYBR-Green Master kit (Roche Diagnostics) was used to perform qPCR. The primers used for the reaction are presented in Table $\mathrm{I}$. The reaction system was the following: 2X SYBR-Green Master Mix (12.5 $\mu \mathrm{l})$; cDNA template $(2 \mu \mathrm{l})$; forward primer $(10 \mu \mathrm{M} ; 1 \mu \mathrm{l})$; reverse primer $(10 \mu \mathrm{M} ; 1 \mu \mathrm{l})$; and $\mathrm{ddH}_{2} \mathrm{O}(8.5 \mu \mathrm{l})$. qPCR was conducted as follows: $95^{\circ} \mathrm{C}$ for $10 \mathrm{~min}$, then 40 cycles of $95^{\circ} \mathrm{C}$ for $15 \mathrm{sec}, 60^{\circ} \mathrm{C}$ for $1 \mathrm{~min}$ and $72^{\circ} \mathrm{C}$ for $3 \mathrm{~min}$. qPCR was conducted in a CFX96 Touch $^{\mathrm{TM}}$ system (cat. no. 6093; Bio-Rad Laboratories, Inc.). The $2^{-\Delta \Delta \mathrm{Cq}}$ method (15) was used to calculate the relative mRNA expression in samples; GAPDH was used as an internal reference.

Extraction of total protein and western blotting. Cells were collected and washed with PBS, and RIPA buffer (Beijing Solarbio Science \& Technology Co., Ltd.) was added to lyse the cells according to the manufacturer's protocols. Cells were then centrifuged at $4^{\circ} \mathrm{C}$ and $16,000 \mathrm{x}$ g for $15 \mathrm{~min}$ to remove the cell debris and supernatant, and total protein was collected. The concentration of total protein was determined using a Pierce ${ }^{\mathrm{TM}}$ BCA Protein Assay kit (Thermo Fisher 
Table I. Primers used for quantitative PCR.

\begin{tabular}{ll}
\hline Genes & \multicolumn{1}{c}{ Primers } \\
\hline SIRT6 & F: 5'-GCAGTCTTCCAGTGTGGTGT-3' \\
& R: 5'-CCATGGTCCAGACTCCGT-3' \\
Bcl-2 & F: 5'-TTGAGGAAGTGAACATTTCGGTG-3' \\
& R: 5'-AGGTTCTGCGGACTTAGGTC-3' \\
Bax & F: 5'-GCGAGTGTCTCAAGCGCATC-3' \\
& R: 5'-CCAGTTGAAGTTGCCGTCAGAA-3' \\
GAPDH & F: 5'-ATGGTGAAGGTCGGTGTGAA-3' \\
& R: 5'-TGGAAGATGGTGATGGGCTT-3'
\end{tabular}

SIRT6, sirtuin 6; F, forward; R, reverse.

Scientific, Inc.). Standard protein was diluted to $1,0.5,0.25$, 0.125 and $0.0625 \mathrm{~g} / \mathrm{ml}$ respectively, then $2 \mu 1$ samples and standard proteins were added to a 96 -well plate. BCA reagent was subsequently added, and plates were incubated at $37^{\circ} \mathrm{C}$ for $30 \mathrm{~min}$. The OD at $562 \mathrm{~nm}$ was detected using a microplate reader. A standard curve was drawn, and the concentration of the total protein was then calculated.

Total protein $(30 \mu \mathrm{g})$ from each sample was denatured at $95^{\circ} \mathrm{C}$ for $10 \mathrm{~min}$, and proteins were separated via $10 \%$ SDS-PAGE at $100 \mathrm{~V}$ for $2 \mathrm{~h}$. Proteins were transferred onto PVDF membranes using a wet transfer electrophoresis tank (Bio-Rad Laboratories, Inc.) at $90 \mathrm{~V}$ for $2 \mathrm{~h}$, and then blocked in $5 \%$ non-fat milk for $1 \mathrm{~h}$ at room temperature. Membranes were then incubated overnight at $4{ }^{\circ} \mathrm{C}$ with the following primary antibodies: Anti-SIRT6 (1:2,000; cat. no. ab191385; Abcam); anti-cleaved-caspase-3 (Asp175; 1:2,000; cat. no. 9664; Cell Signaling Technology, Inc.); anti-Bcl-2 (1:2,000; cat. no. ab32124; Abcam); anti-Bax (1:2,000; cat. no. ab32503; Abcam); anti-GAPDH (1:2,000; cat. no. ab181602; Abcam); anti-ERK1/2 (1:2,000; cat. no. ab17942; Abcam); and anti-phosphorylated (p)-ERK1 (T202) + ERK2 (T185; 1:2,000; cat. no. ab201015; Abcam). Membranes were washed three times with PBS-0.05\% Tween 20 (PBST; Beijing Solarbio Science \& Technology Co., Ltd.) for $5 \mathrm{~min}$, and the horseradish peroxidase-conjugated secondary antibody (1:2,000; cat. no. ab6721; Abcam) was added. Membranes were then incubated for $1 \mathrm{~h}$ at RT, and bands were visualized using Pierce ${ }^{\mathrm{TM}}$ ECL Plus western blotting substrate (Thermo Fisher Scientific, Inc.) following three washes in PBST. The densitometry was performed using the Bio-Rad ChemiDoc system with Image Lab software version 6.0 (Bio-Rad Laboratories, Inc.).

Statistical analysis. Data were analyzed using GraphPad Prism 7.0 software (GraphPad Software, Inc.). All data were presented as the mean \pm standard deviation. One-way ANOVA followed by Tukey's post hoc test were used to compare between groups. $\mathrm{P}<0.05$ was considered to indicate statistical significance.

\section{Results}

SIRT6 is overexpressed in HCC cell lines. To investigate the expression of SIRT6 in HCC cell lines, MIHA, HL7702, Hep3B, Huh-7, MHCC-97H, MHCC-97L, MHCC-LM6, MHCC-LM3,

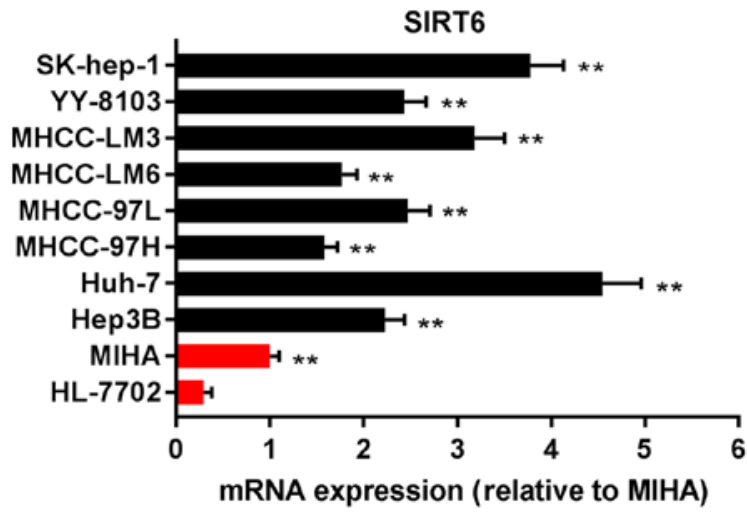

Figure 1. mRNA expression of SIRT6 in HCC cells. The expression of SIRT6 in various HCC and normal cell lines was determined via reverse transcription-quantitative PCR analysis. Data are presented as the mean \pm standard deviation. ${ }^{* *} \mathrm{P}<0.01$ vs. HL-7702. SIRT6, sirtuin 6; HCC, hepatocellular carcinoma.

YY-8103 and SK-hep-1 cell lines were purchased, and the mRNA expression of SIRT6 was measured via RT-qPCR analysis. As presented in Fig. 1, the expression of SIRT6 mRNA in Hep3B, Huh-7, MHCC-97H, MHCC-97L, MHCC-LM6, MHCC-LM3, YY-8103 and SK-hep-1 cells was significantly upregulated compared with HL-7702 cells, normal human liver cells set as the control $(\mathrm{P}<0.01)$. The results indicated that the expression of SIRT6 was elevated in HCC.

Overexpression of SIRT6 increases the proliferation of Huh-7 cells. To investigate the effects of upregulated expression of SIRT6 on the proliferation and apoptosis of HCC cells, the proliferation, cloning efficiency and apoptosis of Huh-7 cells was evaluated following overexpression of SIRT6. Overexpression of SIRT6 mRNA and protein was demonstrated in Huh-7 cells $(\mathrm{P}<0.01$; Fig. 2A and B), and the proliferation of transfected Huh-7 cells was significantly increased compared with the control or NC groups at 24 and $48 \mathrm{~h}$ following transfection $(\mathrm{P}<0.05$; Fig. 2C). Cloning efficiency was increased in the SIRT6 overexpression group compared with the control or NC groups $(\mathrm{P}<0.01$; Fig. $2 \mathrm{E})$; however, the apoptosis rate was not significantly affected by SIRT6 overexpression (Fig. 2D). The results suggested that overexpression of SIRT6 may promote the proliferation of HCC; however, its effects on apoptosis require further investigation.

Knockdown of SIRT6 decreases the proliferation, and increases the apoptosis rate of Huh-7 cells. To further determine the role of SIRT6 in HCC, the effects of silencing SIRT6 on the proliferation and apoptosis of Huh-7 cells was investigated. It was revealed that SIRT6 expression was suppressed in Huh-7 cells following transfection with siSIRT6 $(\mathrm{P}<0.01$; Fig. 3A and B). Additionally, the proliferation of cells in the siSIRT6 group was significantly decreased compared with the control and si-NC groups at 24 and $48 \mathrm{~h}(\mathrm{P}<0.05$; Fig. $3 \mathrm{C})$. Furthermore, the cloning efficiency of siSIRT6-transfected cells was significantly reduced compared with the control and si-NC groups as well $(\mathrm{P}<0.01$; Fig. $3 \mathrm{E})$. The apoptosis rate was also significantly increased in siSIRT6 the group compared with the controls $(\mathrm{P}<0.01$; Fig. 3D). Collectively, the results indicated that the downregulation of SIRT6 
A

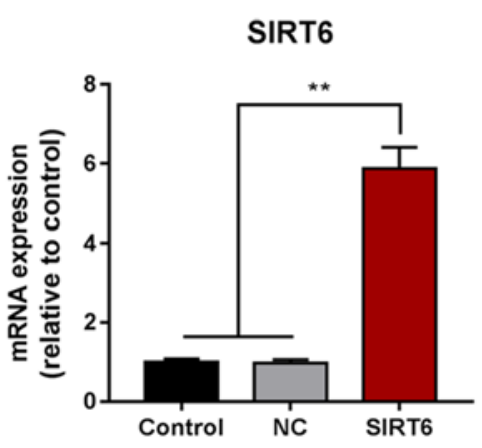

B

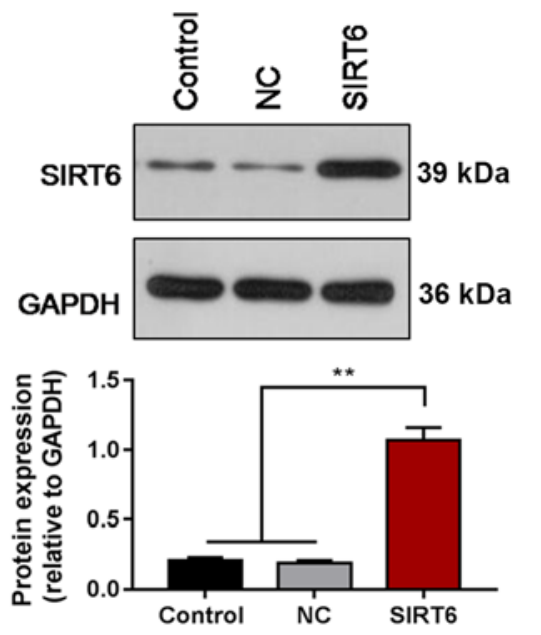

C

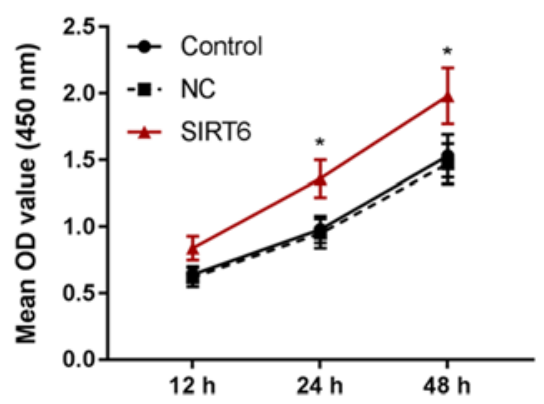

D

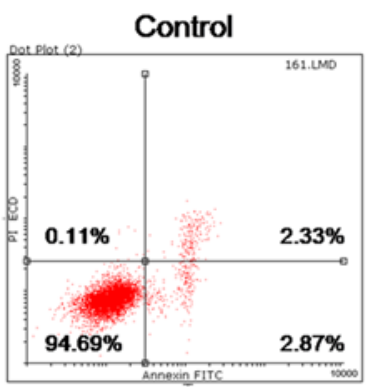

E

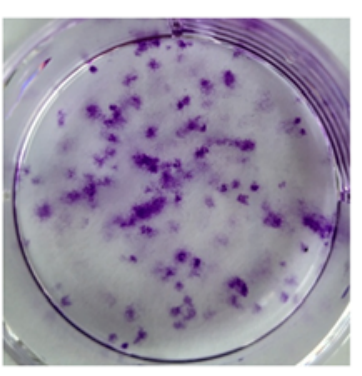

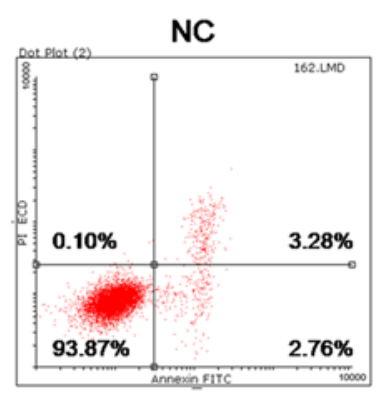
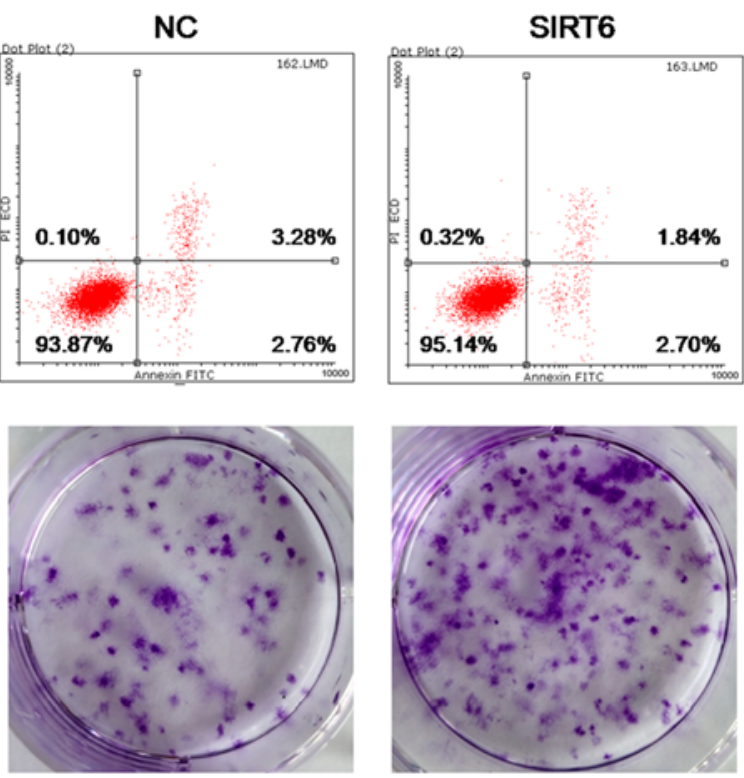
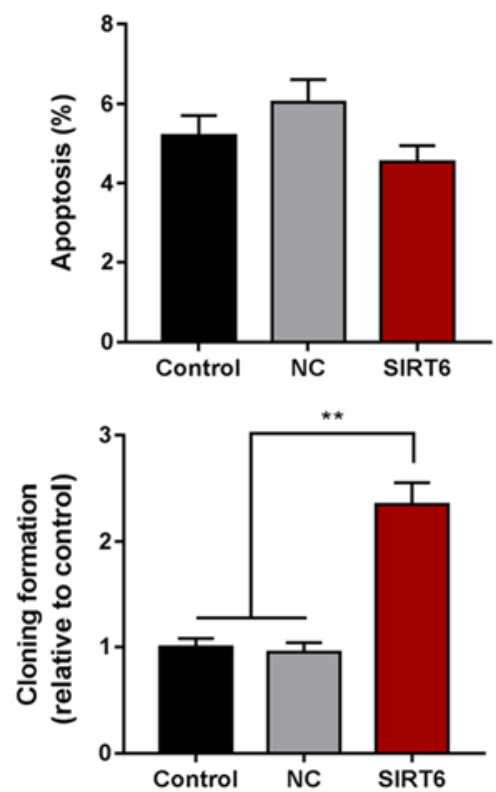

Figure 2. Proliferation, apoptosis and cloning efficiency of Huh-7 cells following overexpression of SIRT6. (A) mRNA expression of SIRT6 as determined via reverse transcription-quantitative PCR analysis. (B) Protein levels of SIRT6 as determined via western blotting. (C) Cell proliferation following transfection for 12, 24 and $48 \mathrm{~h}$ as determined using a Cell Counting Kit-8 assay. (D) Apoptosis of transfected cells as determined via flow cytometry. (E) Clone formation assay and cloning efficiency relative to control. Data are presented as the mean \pm standard deviation. ${ }^{*} \mathrm{P}<0.05,{ }^{* * *} \mathrm{P}<0.01$ vs. control. SIRT6, sirtuin 6; NC, negative control.

decreased the proliferation and increased the apoptosis of HCC cells, opposing effects to the results observed following the overexpression of SIRT6.

Intrinsic apoptosis pathway suppression and activation following SIRT6 overexpression or knockdown, respectively, in Huh-7 cells. To evaluate the activity of the intrinsic apoptosis pathway, the mRNA expression of Bcl-2 and Bax was determined via RT-qPCR analysis, and protein levels of Bcl-2, Bax and cleaved-caspase-3 were measured via western blotting. It was revealed that the expression of Bcl-2 in the SIRT6 overexpression group was significantly upregulated compared with the control or NC groups, and the expression of Bax and cleaved-caspase-3 was significantly downregulated ( $\mathrm{P}<0.05$; Fig. 4A, $\mathrm{C}$ and $\mathrm{E})$. Opposing results were observed following siSIRT6-mediated knockdown of SIRT6 $(\mathrm{P}<0.01$; Fig. 4B, D and F). The results indicated that overexpression of SIRT6 downregulated the intrinsic apoptosis pathway in HCC cells, whereas silencing SIRT6 induced opposing effects.

Activation of the ERK1/2 signal pathway may be involved in the SIRT6-mediated regulation of the proliferation of Huh-7 cells. To investigate the mechanisms underlying the effects of SIRT6 on proliferation and apoptosis, the expression of ERK1/2 and p-ERK1/2 were determined following overexpression or knockdown of SIRT6. Additionally, the role of the ERK1/2 signal pathway in the effects of SIRT6 was explored by measuring the effects of the ERK1/2 inhibitor U0126 on the proliferation of SIRT6-overexpressing Huh-7 cells. It was demonstrated that the phosphorylation of ERK1/2 was significantly increased in the SIRT6 overexpression group $(\mathrm{P}<0.01$; Fig. $4 \mathrm{G}$ and $\mathrm{I})$, and decreased in the siSIRT6 group compared with the control and NC groups $(\mathrm{P}<0.01$; Fig. $4 \mathrm{H}$ and $\mathrm{J})$. Additionally, it was revealed that the proliferation of Huh-7 cells was significantly decreased in 
A

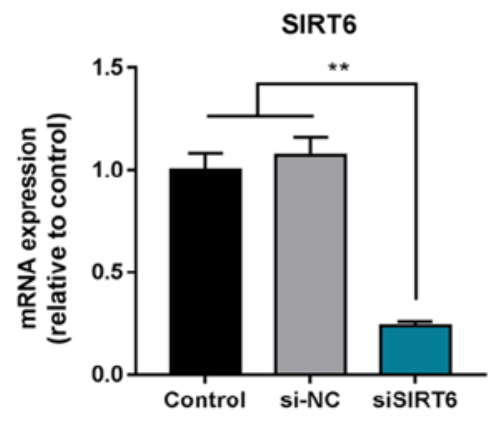

B
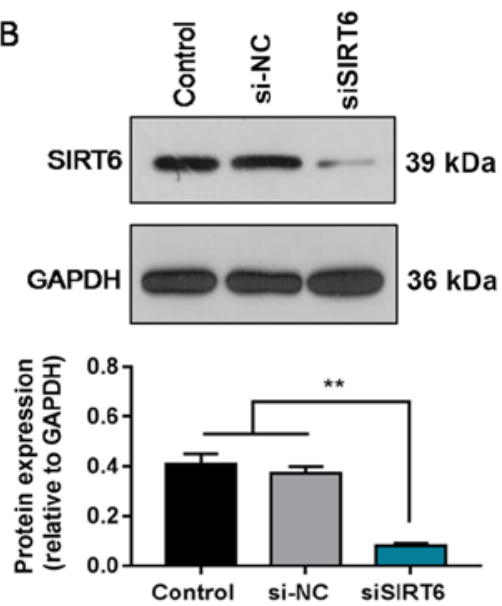

C

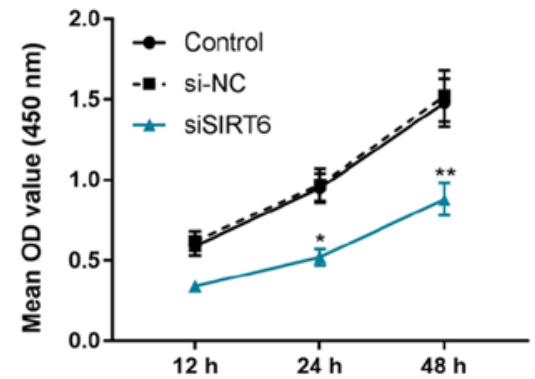

D

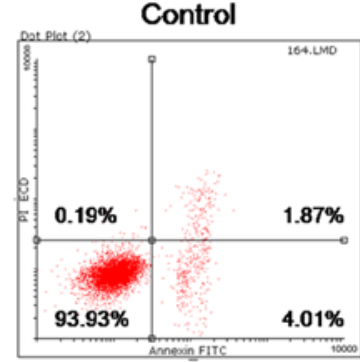

E

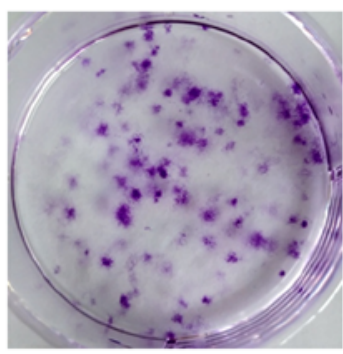

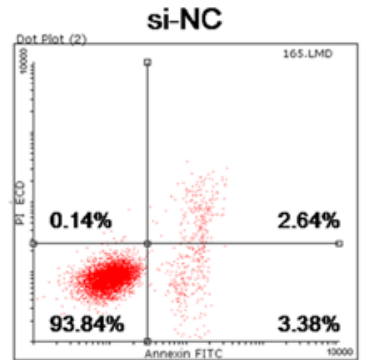
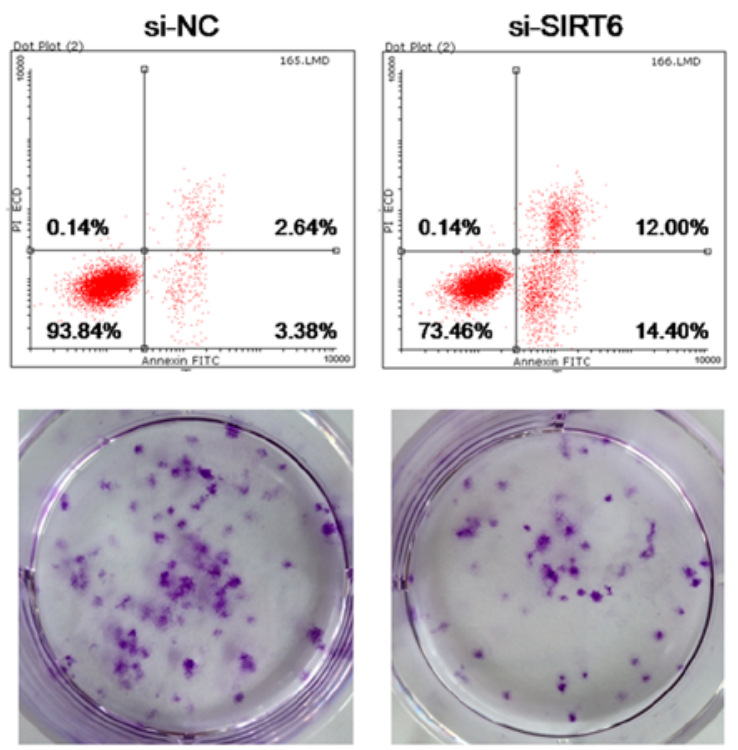
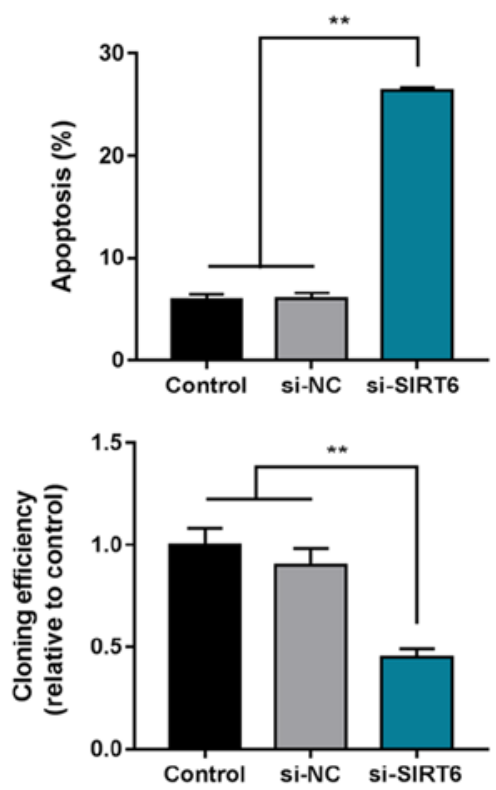

Figure 3. Proliferation, apoptosis and cloning efficiency of Huh-7 cells following knockdown of SIRT6. (A) mRNA expression of SIRT6 as determined via reverse transcription-quantitative PCR analysis. (B) Protein levels of SIRT6 as determined via western blotting. (C) Cell proliferation following transfection for 12, 24 and $48 \mathrm{~h}$ as determined using a Cell Counting Kit-8 assay. (D) Apoptosis of transfected cells as determined via flow cytometry. (E) Clone formation assay and cloning efficiency relative to control. Data are presented as the mean \pm standard deviation. ${ }^{*} \mathrm{P}<0.05,{ }^{* *} \mathrm{P}<0.01$ vs. control. SIRT6, sirtuin 6 ; NC, negative control; siSIRT6, small interfering RNA against SIRT6.

the SIRT6 + U0126 group compared with the SIRT6 group $(\mathrm{P}<0.05)$, and was significantly decreased in the U0126 group compared with the control $(\mathrm{P}<0.05$; Fig. 5). Collectively, the results suggested that the ERK1/2 signal pathway was activated by overexpression of SIRT6 and downregulated by knockdown of SIRT6, and that the ERK1/2 signal pathway may be involved in the SIRT6-mediated regulation of HCC cell proliferation.

\section{Discussion}

In the present study, the expression of SIRT6 was measured in various HCC and normal liver cell lines, and the effects of altering SIRT6 expression on the proliferation and apoptosis of Huh-7 cell lines was evaluated. Furthermore, the involvement of the ERK1/2 signal pathway in the SIRT6-regulated proliferation of Huh-7 cells was investigated, providing novel insight into potential target mechanisms for the treatment of HCC.
The results of the present study demonstrated that SIRT6 was overexpressed in various HCC cell lines. Ran et al (16) also reported that SIRT6 was highly expressed in Huh-7, HepG2, PLC/PRF/5, SMMC-7721, Hep3B and SK-Hep-1 cell lines. Collectively, these studies have demonstrated the overexpression of SIRT6 in a number of HCC cell lines, indicating the value of studying SIRT6 in HCC.

The present study revealed that Huh-7 cell proliferation and cloning efficiency were increased after SIRT6 was overexpressed, and that they were decreased after the SIRT6 was silenced. The apoptosis rate of cells in the SIRT6 overexpression group was not significantly different to those in the control group; however, it was significantly increased following siSIRT6 transfection. Therefore, the results indicated that the expression of SIRT6 was positively associated with the proliferation, and negatively associated with the apoptosis of HCC cells. Feng et al (17) reported that SIRT6 promoted the tumorigenicity of HCC cells. Song et al (18) revealed that following knockout of SIRT6 by CRISPR/Cas-9, HCC cells exhibited decreased viability and 
A

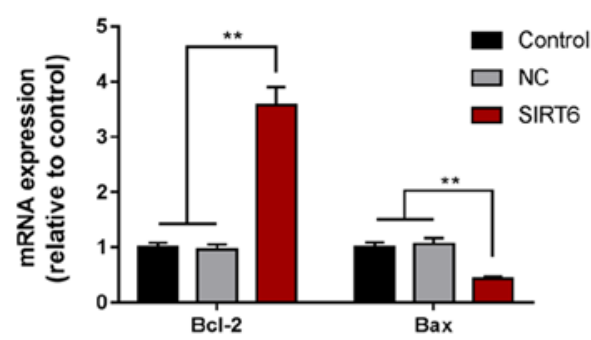

C

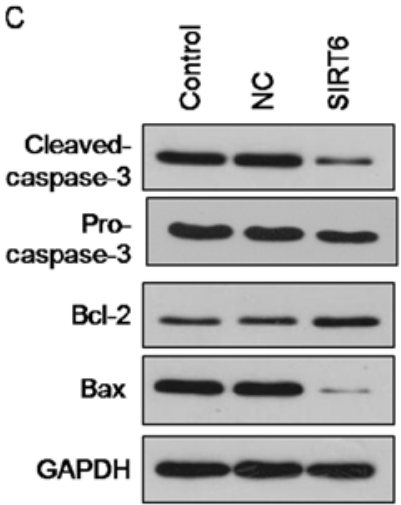

E

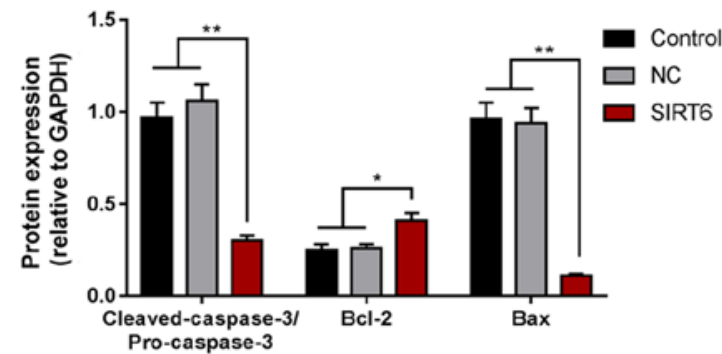

$\mathrm{F}$

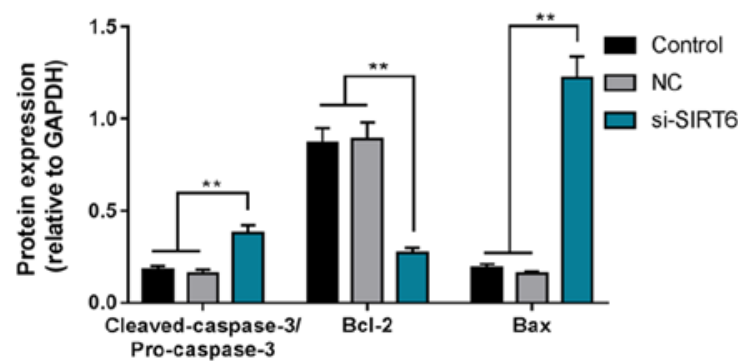

B

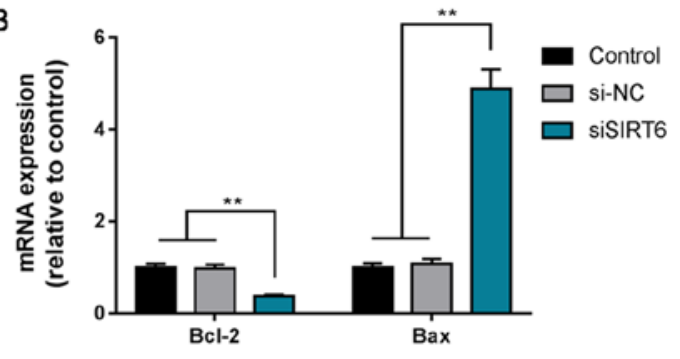

G

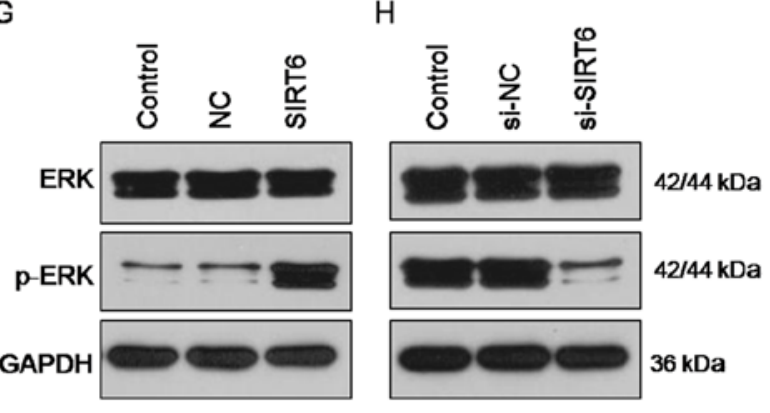

I

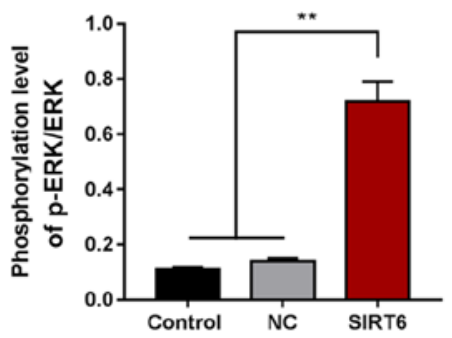

J

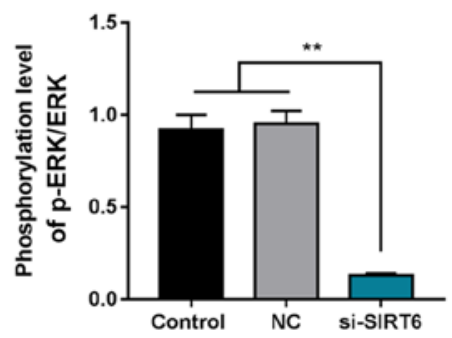

Figure 4. Expression of Bcl-2, Bax, cleaved-caspase-3, ERK1/2, and p-ERK1/2 following overexpression or silencing of SIRT6 in hepatocellular carcinoma cells. (A) mRNA expression of Bcl-2 and Bax in Huh-7 cells following SIRT6 overexpression. (B) mRNA expression of Bcl-2 and Bax in Huh-7 cells following SIRT6 silencing. (C) Protein levels of cleaved-caspase-3, Bcl-2, Bax in Huh-7 cells following SIRT6 overexpression. (D) Protein levels of cleaved-caspase-3, Bcl-2, Bax in Huh-7 cells following SIRT6 silencing. (E) Quantification of (C). (F) Quantification of (D). (G) Protein levels of ERK1/2 and p-ERK1/2 in Huh-7 cells following SIRT6 overexpression. (H) Protein levels of ERK1/2 and p-ERK1/2 in Huh-7 cells following SIRT6 silencing. (I) Quantification of (G), (J) Quantification of $(\mathrm{H})$. Data are presented as the mean \pm standard deviation. ${ }^{*} \mathrm{P}<0.05,{ }^{* *} \mathrm{P}<0.01$. SIRT6, sirtuin 6; NC, negative control; siSIRT6, small interfering RNA against SIRT6; p-, phosphorylated.

invasiveness. These reports are consistent with the observations of the present study; however, Wang et al (19) demonstrated that the overexpression of SIRT6 attenuated HepG2 and HCCLM3 cell proliferation. Similarly, Zhang and Qin (20) reported that knockdown of SIRT6 promoted the growth of HepG2 cells, whereas the overexpression of SIRT6 inhibited HepG2 cell growth. It is proposed that this may be due to SIRT6 performing distinct functions in different HCC cell lines and tumor cells, HepG2 is a hepatoblastoma cell line (21); however, this requires further investigation $(22,23)$.
The intrinsic apoptosis pathway is regulated by the Bcl-2 protein family, and involves mitochondrial outer membrane permeabilization (MOMP) via Bax (24). MOMP results in the release of proapoptotic intermembrane space proteins which ultimately promote apoptosome formation, resulting in caspase- 9 engagement, and caspase- 3 and -7 activation, leading to the apoptosis of cells $(24,25)$. Thus, the expression levels of Bcl-2, Bax and cleaved-caspase- 3 are indicators of the activation of intrinsic apoptosis pathway $(26,27)$. Tumor cells suppress apoptosis via various mechanisms, including 


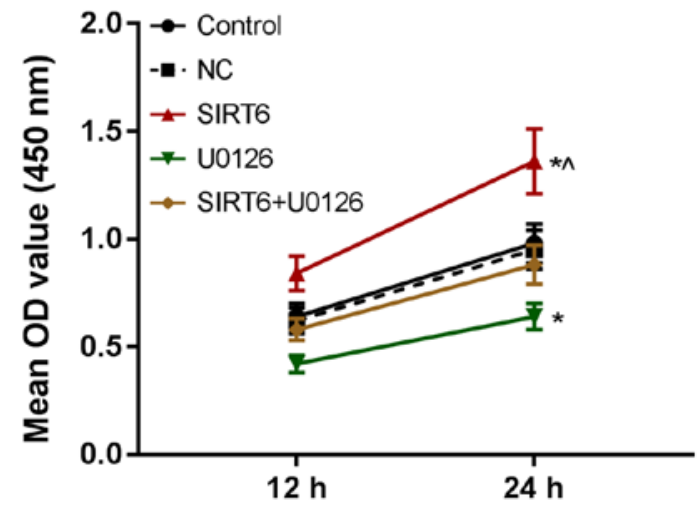

Figure 5. Proliferation of SIRT6-overexpressing hepatocellular carcinoma cells following inhibition of ERK signaling. Proliferation of Huh-7 cells transfected with SIRT6 overexpression plasmid and/or treated with the ERK inhibitor U0126 was determined using a Cell Counting Kit-8 assay. Data are presented as the mean \pm standard deviation. ${ }^{*} \mathrm{P}<0.05$ vs. control ${ }^{\wedge} \mathrm{P}<0.05$ vs. SIRT6 + U0126. SIRT6, sirtuin 6.

promoting the expression of Bcl-2 or downregulating proapoptotic proteins such as Bax $(28,29)$. Sui et al $(30)$ reported that overexpression of Rab31 in Huh-7 cells promoted growth by increasing the $\mathrm{Bcl}-2 / \mathrm{Bax}$ ratio. The present study demonstrated that the expression of Bcl-2 was upregulated, whereas the levels of Bax and cleaved-caspase-3 were downregulated following overexpression of SIRT6, with opposing effects observed following silencing of SIRT6. The results indicated that activation of the intrinsic apoptosis pathway may be negatively associated with the expression of SIRT6 (31).

ERK is a notable serine/threonine protein kinase in the MAPK family (32). Abnormal activation of ERK results in the upregulation of a series of target genes such as matrix metalloproteinase-2 (MMP-2) and vascular endothelial growth factor, promoting the proliferation and invasion of various tumor cells $(33,34)$. The results indicated that the ERK1/2 signal pathway was activated following overexpression of SIRT6 and deactivated by the downregulation of SIRT6. After using the ERK1/2 signal pathway inhibitor U0126, the SIRT6 overexpression-induced increase in Huh-7 cell proliferation was attenuated. Huang et al (35) revealed that paxillin promoted Bcl-2 activation via paxillin-mediated ERK activation, which was associated with tumor formation efficacy in mice in a study of colorectal cancer. Bai et al (12) also reported that the overexpression of SIRT6 promoted the migration and invasion of non-small cell lung cancer cells via the ERK1/2/MMP-9 pathway. These reports indicated that overexpression of SIRT6 can activate the ERK1/2 pathway and therefore suppresses the intrinsic apoptosis pathway, promoting the development of HCC. Conversely, Wang et al (19) observed that the overexpression of SIRT6 reduced the expression of p-ERK in HepG2 and HCCLM3 cells. Zhang and Qin (20) revealed that overexpression of SIRT6 inhibited ERK1/2, and that inhibiting the pathway with U0126 attenuated the tumor-suppressive effects of SIRT6 overexpression. Thus, further investigation is required to determine the precise roles of SIRT6 and the ERK1/2 signaling pathway in HCC.

SIRT6 is an NAD(+)-dependent deacetylase. A previous study identified SIRT6 protein as a potential drug target (36).
Progress has been made in the study of the SIRT6 structure and its inhibitors, and certain small molecules with improved inhibition of the biological functions of STIR6 have been discovered (37-39). The present findings suggested that SIRT6 is a potential target for the treatment of liver cancer; however, this study also possessed certain shortcomings. Huh-7 cells were selected for further experiments as they exhibited the highest SIRT6 expression out of the $8 \mathrm{HCC}$ cell lines; however, these proliferation and apoptosis results can be more fully demonstrated if multiple HCC cell lines were used. Animal experiments should be conducted in future studies to validate the present findings in vivo. In addition, ERK is involved in the transduction of numerous signaling pathways, including the MAPK,PI3K/AKT and STAT pathways. It was demonstrated that SIRT6 contributed to tumor development by regulating ERK phosphorylation; the downstream mechanisms should be explored in subsequent studies.

In conclusion, SIRT6 regulated the proliferation and apoptosis of HCC cells via the regulation of the ERK1/2 pathway, affecting the activation of the intrinsic apoptosis pathway. The present study revealed that SIRT6 may be a potential target in the gene therapy of HCC; however, the role of SIRT6 in HCC requires further validation.

\section{Acknowledgements}

Not applicable.

\section{Funding}

No funding was received.

\section{Availability of data and materials}

The analyzed data sets generated during the study are available from the corresponding author on reasonable request.

\section{Authors' contributions}

CZ, YY and KT made substantial contributions to the conception and design of the study. QH and KT was involved in data acquisition, data analysis and interpretation. CZ and YY drafted the manuscript and critically revised it. All authors gave final approval of the version to be published, and agreed to be accountable for all aspects of the work in ensuring that questions related to the accuracy or integrity of the work are appropriately investigated and resolved.

\section{Ethics approval and consent to participate}

Not applicable.

\section{Patient consent for publication}

Not applicable.

\section{Competing interests}

The authors declare that they have no competing interests. 


\section{References}

1. Torre LA, Bray F, Siegel RL, Ferlay J, Lortet-Tieulent J and Jemal A: Global cancer statistics, 2012. CA Cancer J Clin 65: 87-108, 2015

2. Kennedy AS and Sangro B: Nonsurgical treatment for localized hepatocellular carcinoma. Curr Oncol Rep 16: 373, 2014

3. Hanahan D and Weinberg RA: Hallmarks of cancer: The next generation. Cell 144: 646-674, 2011.

4. Blander G and Guarente L: The Sir2 family of protein deacetylases. Annu Rev Biochem 73: 417-435, 2004.

5. Vaquero A, Scher M, Lee D, Erdjument-Bromage H, Tempst P and Reinberg D: Human SirT1 interacts with histone H1 and promotes formation of facultative heterochromatin. Mol Cell 16: 93-105, 2004.

6. Huang G, Hao F and Hu X: Downregulation of microRNA-155 stimulates sevoflurane-mediated cardioprotection against myocardial ischemia/reperfusion injury by binding to SIRT1 in mice. J Cell Biochem, 2019.

7. Zhang HX, Li YN, Wang XL, Ye CL, Zhu XY, Li HP, Yang T and Liu YJ: Probucol ameliorates EMT and lung fibrosis through restoration of SIRT3 expression. Pulm Pharmacol Ther: 101803, 2019.

8. Jeong SG and Cho GW: The tubulin deacetylase sirtuin-2 regulates neuronal differentiation through the ERK/CREB signaling pathway. Biochem Biophys Res Commun 482: 182-187, 2017.

9. Ming M, Han W, Zhao B, Sundaresan NR, Deng CX, Gupta MP and He YY: SIRT6 promotes COX-2 expression and acts as an oncogene in skin cancer. Cancer Res 74: 5925-5933, 2014.

10. Vitiello M, Zullo A, Servillo L, Mancini FP, Borriello A, Giovane A, Della Ragione F, D'Onofrio N and Balestrieri ML: Multiple pathways of SIRT6 at the crossroads in the control of longevity, cancer, and cardiovascular diseases. Ageing Res Rev 35: 301-311, 2017.

11. Liu Y, Xie QR, Wang B, Shao J, Zhang T, Liu T, Huang G and $\mathrm{Xia} \mathrm{W}$ : Inhibition of SIRT6 in prostate cancer reduces cell viability and increases sensitivity to chemotherapeutics. Protein Cell 4: 702-710, 2013.

12. Bai L, Lin G, Sun L, Liu Y, Huang X, Cao C, Guo Y and Xie C: Upregulation of SIRT6 predicts poor prognosis and promotes metastasis of non-small cell lung cancer via the ERK1/2/MMP9 pathway. Oncotarget 7: 40377-40386, 2016.

13. Li L, Zhao GD, Shi Z, Qi LL, Zhou LY and Fu ZX: The Ras/Raf/MEK/ERK signaling pathway and its role in the occurrence and development of HCC. Oncol Lett 12: 3045-3050, 2016.

14. Ni Z, Wang B, Dai X, Ding W, Yang T, Li X, Lewin S, Xu L, Lian J and He F: HCC cells with high levels of Bcl-2 are resistant to ABT-737 via activation of the ROS-JNK-autophagy pathway. Free Radic Biol Med 70: 194-203, 2014.

15. Livak KJ and Schmittgen TD: Analysis of relative gene expression data using real-time quantitative PCR and the 2(-Delta Delta C(T)) method. Methods 25: 402-408, 2001.

16. Ran LK, Chen Y, Zhang ZZ, Tao NN, Ren JH, Zhou L, Tang H, Chen X, Chen K, Li WY, et al: SIRT6 overexpression potentiates apoptosis evasion in hepatocellular carcinoma via BCL2-associated X protein-dependent apoptotic pathway. Clin Cancer Res 22: 3372-3382, 2016.

17. Feng XX, Luo J, Liu M, Yan W, Zhou ZZ, Xia YJ, Tu W, Li PY, Feng $\mathrm{ZH}$ and Tian DA: Sirtuin 6 promotes transforming growth factor- $\beta 1 / \mathrm{H} 2 \mathrm{O} 2 / \mathrm{HOCl}$-mediated enhancement of hepatocellular carcinoma cell tumorigenicity by suppressing cellular senescence. Cancer Sci 106: 559-566, 2015.

18. Song S, Yang Y, Liu M, Liu B, Yang X, Yu M, Qi H, Ren M, Wang Z, Zou J, et al: MiR-125b attenuates human hepatocellular carcinoma malignancy through targeting SIRT6. Am J Cancer Res 8: 993-1007, 2018.

19. Wang Y, Pan T, Wang H, Li L, Li J, Zhang D and Yang H: Overexpression of SIRT6 attenuates the tumorigenicity of hepatocellular carcinoma cells. Oncotarget 8: 76223-76230, 2017.

20. Zhang ZG and Qin CY: Sirt6 suppresses hepatocellular carcinoma cell growth via inhibiting the extracellular signal-regulated kinase signaling pathway. Mol Med Rep 9: 882-888, 2014.
21. López-Terrada D, Cheung SW, Finegold MJ and Knowles BB: Hep G2 is a hepatoblastoma-derived cell line. Hum Pathol 40: $1512-1515,2009$

22. Etchegaray JP, Zhong L and Mostoslavsky R: The histone deacetylase SIRT6: At the crossroads between epigenetics, metabolism and disease. Curr Top Med Chem 13: 2991-3000, 2013.

23. Lerrer B, Gertler AA and Cohen HY: The complex role of SIRT6 in carcinogenesis. Carcinogenesis 37: 108-118, 2016.

24. Kalkavan H and Green DR: MOMP, cell suicide as a BCL-2 family business. Cell Death Differ 25: 46-55, 2018.

25. Green DR and Llambi F: Cell death signaling. Cold Spring Harb Perspect Biol 7, 2015.

26. Khodapasand E, Jafarzadeh N, Farrokhi F, Kamalidehghan B and Houshmand $\mathrm{M}$ : Is $\mathrm{Bax} / \mathrm{Bcl}-2$ ratio considered as a prognostic marker with age and tumor location in colorectal cancer? Iran Biomed J 19: 69-75, 2015.

27. Pastor-Idoate S, Rodríguez-Hernández I, Rojas J, Fernández I, Garcia-Gutierrez MT, Ruiz-Moreno JM, Rocha-Sousa A, Ramkissoon YD, Harsum S, MacLaren RE, et al: BAX and BCL-2 polymorphisms, as predictors of proliferative vitreoretinopathy development in patients suffering retinal detachment: The Retina 4 project. Acta Ophthalmol 93: e541-e549, 2015.

28. Hassan M, Watari H, AbuAlmaaty A, Ohba Y and Sakuragi N: Apoptosis and molecular targeting therapy in cancer. Biomed Res Int 2014: 150845, 2014.

29. Goldar S, Khaniani MS, Derakhshan SM and Baradaran B: Molecular mechanisms of apoptosis and roles in cancer development and treatment. Asian Pac J Cancer Prev 16: 2129-2144, 2015.

30. Sui Y, Zheng X and Zhao D: Rab31 promoted hepatocellular carcinoma (HCC) progression via inhibition of cell apoptosis induced by PI3K/AKT/Bcl-2/BAX pathway. Tumour Biol 36: 8661-8670, 2015

31. Morigi M, Perico L and Benigni A: Sirtuins in renal health and disease. J Am Soc Nephrol 29: 1799-1809, 2018.

32. Xing X, Gu X, Ma T and Ye H: Biglycan up-regulated vascular endothelial growth factor (VEGF) expression and promoted angiogenesis in colon cancer. Tumour Biol 36: 1773-1780, 2015.

33. Miyake M, Goodison S, Lawton A, Gomes-Giacoia E and Rosser CJ: Angiogenin promotes tumoral growth and angiogenesis by regulating matrix metallopeptidase- 2 expression via the ERK1/2 pathway. Oncogene 34: 890-901, 2015.

34. Sun Y, Liu WZ, Liu T, Feng X, Yang N and Zhou HF: Signaling pathway of MAPK/ERK in cell proliferation, differentiation, migration, senescence and apoptosis. J Recept Signal Transduct Res 35: 600-604, 2015.

35. Huang CC, Wu DW, Lin PL and Lee H: Paxillin promotes colorectal tumor invasion and poor patient outcomes via ERK-mediated stabilization of Bcl-2 protein by phosphorylation at Serine 87. Oncotarget 6: 8698-8708, 2015.

36. Kim JH, Lee JM, Kim JH and Kim KR: Fluvastatin activates sirtuin 6 to regulate sterol regulatory element-binding proteins and AMP-activated protein kinase in HepG2 cells. Biochem Biophys Res Commun 503: 1415-1421, 2018.

37. Parenti MD, Grozio A, Bauer I, Galeno L, Damonte P, Millo E, Sociali G, Franceschi C, Ballestrero A, Bruzzone S, et al: Discovery of novel and selective SIRT6 inhibitors. J Med Chem 57: 4796-4804, 2014.

38. Sociali G, Galeno L, Parenti MD, Grozio A, Bauer I, Passalacqua M, Boero S, Donadini A, Millo E, Bellotti M, et al: Quinazolinedione SIRT6 inhibitors sensitize cancer cells to chemotherapeutics. Eur J Med Chem 102: 530-539, 2015.

39. Damonte P, Sociali G, Parenti MD, Soncini D, Bauer I, Boero S, Grozio A, Holtey MV, Piacente F, Becherini P, et al: SIRT6 inhibitors with salicylate-like structure show immunosuppressive and chemosensitizing effects. Bioorg Med Chem 25: 5849-5858, 2017.

This work is licensed under a Creative Commons Attribution-NonCommercial-NoDerivatives 4.0 International (CC BY-NC-ND 4.0) License. 